\title{
Análise Termogravimétrica do Efeito da Granulometria do Resíduo Metalúrgico na Cinética de Perda de Massa
}

\section{Thermogravimetric analysis of the granulometric effect of metallurgical residue in the kinetics of mass loss}

\author{
1 Manara Nogueira Quintino mnquintino@gmail.com \\ 1 José Adilson de Castro \\ 1 Leonardo Martins da Silva \\ 1 Izabel de Oliveira Mota
}

1 Universidade Federal Fluminense

\section{RESUMO}

No processo de produção de ferro e aço, uma grande quantidade de resíduos é gerada. Esses resíduos e co-produtos contêm consideráveis quantidades de ferro e carbono, dentre outros elementos em menores concentrações, que podem ser reaproveitados, visando reduzir o impacto ambiental proveniente do descarte inadequado. 0 objetivo principal deste trabalho foi analisar a perda de massa de uma mistura de carvão vegetal, capim elefante e resíduo metalúrgico sob ação de um fluxo de gás inerte por meio de análise termogravimétrica. 0 tamanho de grão do resíduo foi alterado, a fim de se analisar o impacto dessa variável no processo de perda de massa. Com o propósito de se explicar o processo catalítico, cálculos cinéticos foram realizados, utilizando-se o método Coats-Redfern considerando reações de primeira ordem. Pode-se observar que um menor tamanho de grão possibilita uma menor quantidade residual ao final do experimento, o que foi confirmado pelos cálculos cinéticos realizados.

\section{Palavras-chave}

Análise termogravimétrica. Biomassa. Resíduo metalúrgico.

\begin{abstract}
During iron and steel making process, a large residue amount is generated. This residue and its co products contain considerable quantities of iron and carbon, among other elements at lower concentrations. They can be reused aiming the decrease of environmental pollution that comes with the inappropriate discard. The main aim of this study was evaluate the mass loss of a mixture of charcoal, elephant grass biomass and metallurgical residue under the action of an inert gas flow through thermogravimetric analysis. The grain size of the residue was altered in order to analyze the impact of this variable in the mass loss process. In order to explain the catalytic process, kinetic calculations were performed using the Coats-Redfern method considering first order reactions. It was observed that a smaller grain size allows a smaller residual mass at the end of the experiment, which was confirmed by the kinetic calculations performed.
\end{abstract}

\section{Keywords}

Thermogravimetric analysis. Biomass. Metallurgical residues.

\section{Como você deve citar?}

QUINTINO, Manara Nogueira et al. Análise Termogravimétrica do Efeito da Granulometria do Resíduo Metalúrgico na Cinética de Perda de Massa. Cadernos UniFOA, Volta Redonda, n. 35, p. 5-13, dez. 2017. 
Análise Termogravimétrica do Efeito da Granulometria do

Resíduo Metalúrgico na Cinética de Perda de Massa

\section{INTRODUÇÃO}

A população está cada vez mais interessada nos impactos ambientais da produção, uso e descarte de produtos, assim como as normas para a reutilização e descarte dos resíduos e co-produtos gerados se tornaram mais severas ao longo dos anos. Devido a isso, os responsáveis devem encaminhá-los para o reuso e descarte adequado [1].

O setor metalúrgico é um grande produtor de resíduos e co-produtos, tendo produzido, em 2013, cerca de 17 milhões de toneladas desses materiais [2]. Resíduos ricos em ferro, como poeiras e cinzas de alto-forno normalmente, são reaproveitados no próprio setor siderúrgico por meio de sinterização ou pelotização, porém essas não são as práticas mais indicadas para o reaproveitamento desse tipo de resíduo, pois aumenta o consumo de energia e reduz a permeabilidade do leito [3, 4].

Estudos ao longo dos anos têm sido realizados com o intuito de melhorar a eficiência de combustão do carvão, quando misturado a resíduos e/ou componentes metálicos [4]. Li et al. [5], investigaram os efeitos catalíticos e de aceleração de óxidos metálicos ( $\mathrm{CuO}, \mathrm{Fe}_{2} \mathrm{O}_{3}$ e $\mathrm{ZnO}$ ) nas características de ignição e combustão do carvão utilizando TGA. Os autores observaram que as diferenças entre as taxas de combustão máximas e as correspondentes temperaturas (temperatura de pico) das amostras com e sem os óxidos metálicos puderam ser atribuídas aos efeitos catalíticos e de aceleração dos óxidos metálicos no processo de combustão assim como as diferenças nas temperaturas de ignição indicam que os óxidos metálicos usados podem acelerar e catalisar a ignição do carvão.

Gong et. al. [6] investigaram os efeitos do $\mathrm{CeO}_{2}$ e $\mathrm{Fe}_{2} \mathrm{O}_{3}$ como catalisadores da combustão de carvão pulverizado, e compararam as amostras com e sem a presença de catalisador na reação de combustão. Os autores observaram que o indicador de combustão total mostrou que a mistura de carvão com ambos os catalisadores foi maior que aqueles puros, mostrando que ambos os aditivos melhoraram a reação de combustão desses combustíveis e aceleraram a combustão de carbono fixo.

Zou e Zhao [4] estudaram o comportamento de materiais em pó que continham ferro (hematita, pó de alto-forno, pó de conversor de aciaria e lama de aciaria) no comportamento da combustão do carvão por meio de análise termogravimétrica. Os autores concluíram que a hematita, pó e lama de aciaria conseguiram melhorar o comportamento da combustão total. Contudo, a morfologia se mostrou mais importante do que a composição química nos efeitos catalíticos dos aditivos na combustão do carvão.

A Análise Térmica da Cinética (Thermal Analysis Kinetics - TAK) busca analisar quantitativamente as relações entre temperatura e propriedades físicas, por exemplo, mudança de massa em função do tempo, medidas por técnicas de análise térmica [7]. As técnicas mais comumente utilizadas para a obtenção de parâmetros cinéticos são TGA e DSC. Através da análise desses resultados, a TAK é capaz de estimar a estabilidade térmica e vida útil dos materiais, descrever quantitativamente a taxa e os mecanismos de reação e fornecer informações de suporte para estimar propriedades de materiais energéticos e combustíveis. Tais ferramentas também têm se mostrado efetivas para o estudo dos parâmetros característicos da combustão do carvão, sendo largamente aplicados para o estudo da catálise dessa reação [4 - 6, 8 - 12].

O objetivo deste estudo é analisar a perda de massa de uma mistura de carvão vegetal, capim elefante e resíduo metalúrgico sob ação de um fluxo de gás inerte, uma vez que os óxidos metálicos presentes no resíduo servem como fornecedores de oxigênio. 0 tamanho de grão do resíduo é alterado, a fim de se analisar o impacto dessa variável no processo de perda de massa. Com o propósito de se explicar o processo catalítico, cálculos cinéticos foram realizados utilizando-se o método Coats-Redfern considerando reações de primeira ordem. 


\section{MATERIAIS E MÉTODOS}

Dois tipos de biomassa foram utilizados nesta pesquisa: o carvão vegetal e carvão de capim elefante. 0 resíduo metalúrgico utilizado, o pó de alto-forno, foi cedido por uma indústria siderúrgica localizada na região sudeste do Brasil. Como pode ser observado na Tabela 1, o qual apresenta a composição do resíduo, este apresenta elevada concentração de óxido de ferro e carbono.

Tabela 1: Análise Química do Pó de Alto Forno

\begin{tabular}{|c|c|c|c|c|c|c|c|c|c|c|c|c|c|c|}
\hline $\begin{array}{l}\text { MATERIAL } \\
(\%)\end{array}$ & $\begin{array}{c}\mathrm{Fe} \\
\text { TOTAL }\end{array}$ & $\mathrm{Fe}_{2} \mathrm{O}_{3}$ & $\mathrm{FeO}$ & $\mathrm{Fe}_{\text {metálico }}$ & $\mathrm{SiO}_{2}$ & $\mathrm{Al}_{2} \mathrm{O}_{3}$ & MgO & C & $\mathrm{CaO}$ & $\mathrm{Na}_{2} \mathrm{O}$ & $\mathrm{SO}_{3}$ & $\mathrm{~Pb}$ & $\mathrm{P}_{2} \mathrm{O}_{5}$ & $\mathrm{ZnO}$ \\
\hline PÓ DE & & & & & & & & & & & & & & \\
\hline $\begin{array}{c}\text { COLETOR } \\
\text { DE AF\#3 }\end{array}$ & 33,88 & 37,49 & 8,05 & 1,34 & 10,29 & 2,66 & 2,19 & 30,57 & 3,3 & 0,047 & 0,678 & 0,041 & 0,078 & 0,484 \\
\hline
\end{tabular}

Fonte: os autores, 2017

Esses materiais foram escolhidos por serem finos, conterem alta reatividade e usualmente necessitarem de estar aglomerados para a análise.

O resíduo foi peneirado em peneiras vibratórias e a fração passante utilizada foi: 0,044mm até $0,088 \mathrm{~mm}$ e $0,125 \mathrm{~mm}$ até $0,210 \mathrm{~mm}$. As porções utilizadas de carvão vegetal, capim elefante e resíduo metalúrgico estão indicadas na Tabela 2. As amostras foram pesadas em balança de precisão, diretamente no cadinho.

Tabela 2: Matriz de Planejamento das Misturas

\begin{tabular}{|l|l|l|l|l|}
\hline Amostra & Resíduo (\%) & $\begin{array}{l}\text { Capim Elefante } \\
(\%)\end{array}$ & Carvão Vegetal (\%) & Granulometria (mm) \\
\hline 1 & 6 & 12,5 & 81,5 & $0,044-0,088$ \\
\hline 2 & 6 & 12,5 & 81,5 & $0,125-0,210$ \\
\hline
\end{tabular}

Fonte: os autores, 2017

Para a avaliação da potencialidade de aquecimento dos resíduos siderúrgicos foram realizados experimentos com a finalidade de se analisar a cinética de algumas reações que acontecem no processo de combustão. Para essa análise, foi utilizado o equipamento de TGA Q50 da TA Instruments, com amostras com peso $4 \pm 0,1 \mathrm{mg}$, com uma taxa de aquecimento de $15^{\circ} \mathrm{C} / \mathrm{min}$. 0 intervalo de temperatura de aquecimento foi da temperatura ambiente até $800^{\circ} \mathrm{C}$, com um fluxo de gás de $50 \mathrm{~mL} / \mathrm{min}$ em uma atmosfera de nitrogênio. Após atingir a temperatura máxima, a temperatura permaneceu constante por $25 \mathrm{~min}$.

Com os resultados dos experimentos, obteve-se a perda de massa, energia de ativação aparente, fator pré-exponencial e taxa de conversão.

Para o cálculo das constantes cinéticas, utilizou-se o método de Coats-Redfern para calcular a energia de ativação $E$ e o fator pré-exponencial $A$, com base nas curvas de TGA [5]. Esse método baseia-se no fato de que as reações de combustão sejam reações de primeira ordem.

Usualmente, para os estudos cinéticos, a taxa de conversão é expressa pela seguinte equação: 


$$
\frac{d \alpha}{d t}=k f(\alpha)
$$

Essa equação representa a taxa de conversão, $d \alpha / d t$, a uma temperatura constante em função da perda de massa do reagente e da constante de velocidade $k$, que representa a constante da taxa de reação, sendo $f(\alpha)$ um modelo de mecanismo de reação. A conversão de $\alpha$ é definida pela equação 2 :

$$
\alpha=\frac{m_{0}-m}{m_{0}-m_{f}}
$$

Onde $m$ representa a massa do reagente e os subscritos 0 e $f$ representam a quantidade no início e no fim do experimento, respectivamente. A constante $k$ é calculada, utilizando-se a expressão de Arrhenius:

$$
k(T)=A \exp \left(-\frac{E}{R T}\right)
$$

Combinando as equações 1 e 3, a taxa de reação pode ser escrita da seguinte maneira:

$$
\frac{d \alpha}{d t}=\operatorname{Aexp}\left(-\frac{E}{R T}\right) f(\alpha)
$$

Para a combustão do carvão:

$$
f(\alpha)=(1-\alpha)
$$

Para processos não isotérmicos, como os estudados com o auxilio do TGA, substituindo a taxa de aquecimento $\beta$, onde:

$$
\beta=d T / d t
$$

Chega-se a seguinte expressão:

$$
\frac{d \alpha}{d T}=\frac{A}{\beta} \exp \left(-\frac{E}{R T}\right)(1-\alpha)
$$

A solução da integral da equação 7 é:

$$
\ln \left(-\frac{\ln (1-\alpha)}{T^{2}}\right)=\ln \left(\frac{A R}{\beta E}\right)-\frac{E}{R T}
$$

Com base no resultado do gráfico de $\ln \left[-\ln (1-a) / T^{2}\right]$ versus $100 / T$, utiliza-se a regressão linear para calcular os valores de E e A. 


\section{ANÁLISE E DISCUSSÕES}

A Figura 1 apresenta o perfil de perda de massa em função do tempo para as amostras nas duas diferentes granulometrias.

Figura 1: Curvas dos ensaios de TGA

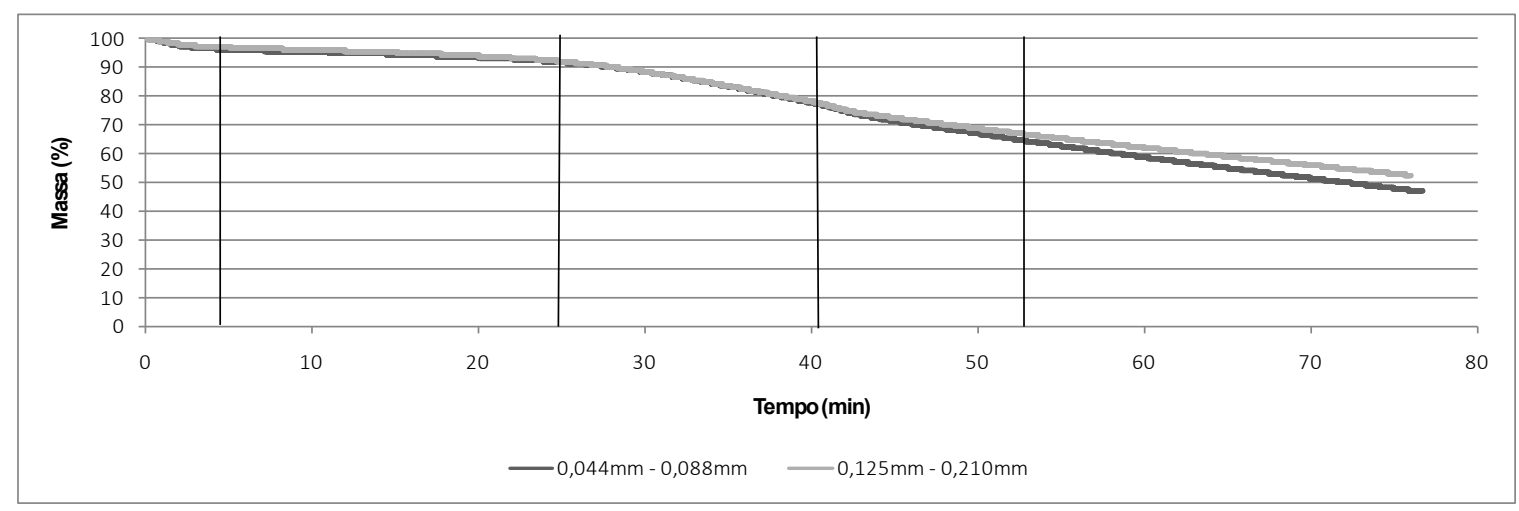

Fonte: os autores, 2017

As propriedades térmicas do resíduo estão baseadas nos dados obtidos experimentalmente durante todo o teste, incluindo a fase isotérmica. Percebe-se que há uma perda de massa inicial, entre $25^{\circ} \mathrm{C}$ e $100^{\circ} \mathrm{C}$ (primeiros $5 \mathrm{~min}$ de experimento), sendo esta devido à perda de umidade contida na amostra, conforme o trabalho de Jian et. al. [13].

Nota-se que o formato da curva é bem similar para ambas as amostras, ocorrendo vários pontos de intersecção até cerca de $40 \mathrm{~min}$ de experimento. Após esse período, a amostra que possui menor tamanho de grão passa a perder massa mais rapidamente, ocorrendo a maior diferença em porcentagem de perda ao final do experimento, com $46,7 \%$ de massa residual para a amostra com granulometria entre 0,044 a $0,088 \mathrm{~mm}$ e $52,5 \%$ para a amostra com granulometria entre 0,125 a $0,210 \mathrm{~mm}$.

As Figuras 2 e 3 apresentam as curvas de taxa de conversão experimentais, calculadas para as amostras com granulometria entre 0,044 a 0,088 $\mathrm{mm}$ e 0,125 a 0,210 $\mathrm{mm}$ respectivamente. A curva de taxa foi feita utilizando-se o método Coats-Redfern, considerando reação de primeira ordem. Além disso, foram selecionadas diferentes faixas de tempo, a fim de se aproximar ao máximo possível a curva experimental e calculada. A partir das diferentes faixas de temperatura, foi possível realizar o cálculo cinético e assim se obter as constantes cinéticas nas diferentes faixas de temperatura. 
Análise Termogravimétrica do Efeito da Granulometria do

Resíduo Metalúrgico na Cinética de Perda de Massa

Figura 2 - Curvas de conversão para a amostra com granulometria 0,044-0,088mm

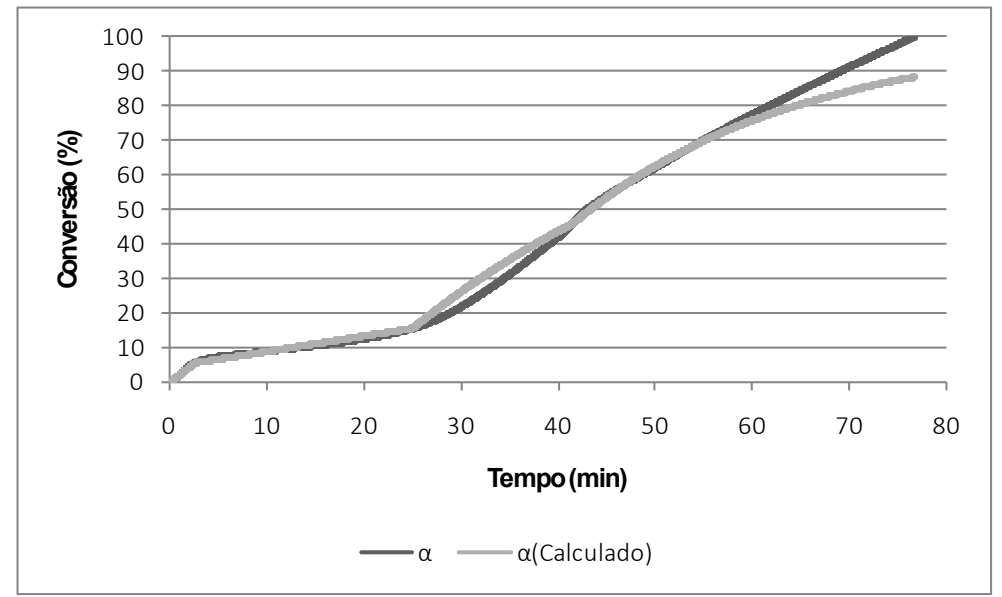

Fonte: os autores, 2017

Figura 3 - Curvas de conversão para a amostra com granulometria 0,125-0,210

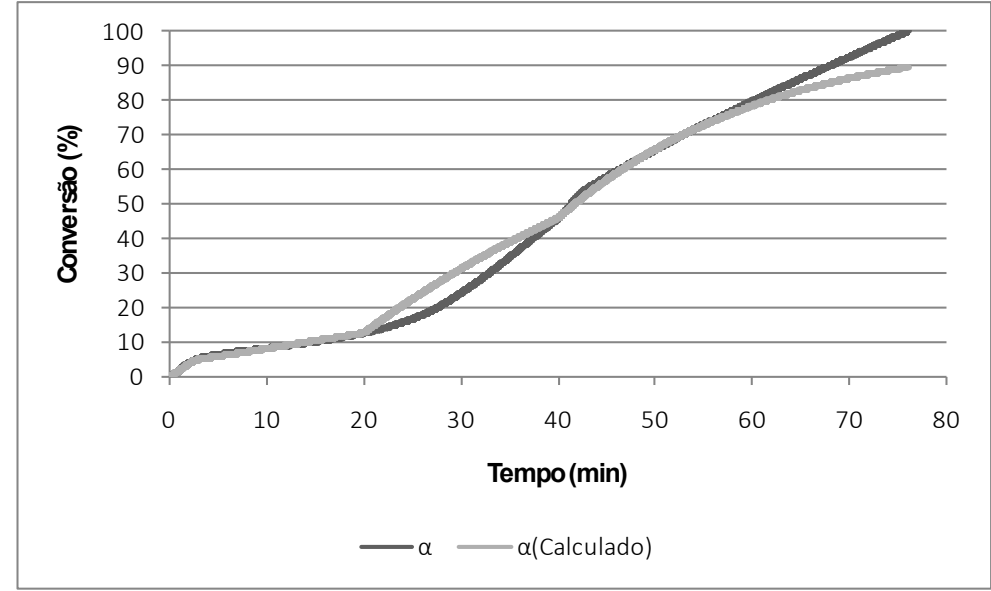

Fonte: os autores, 2017

A Figura 4 apresenta as curvas de conversão para as amostras nas diferentes granulometrias. Assim como ocorre nas curvas de TGA analisadas previamente, percebe-se que a taxa de queima de massa para ambas as amostras é semelhante, ocorrendo intersecções entre as curvas em vários pontos do gráfico. Os valores cinéticos calculados para as amostras são apresentados na Tabela 3. 
Figura 4 - Curvas de Conversão para as amostras

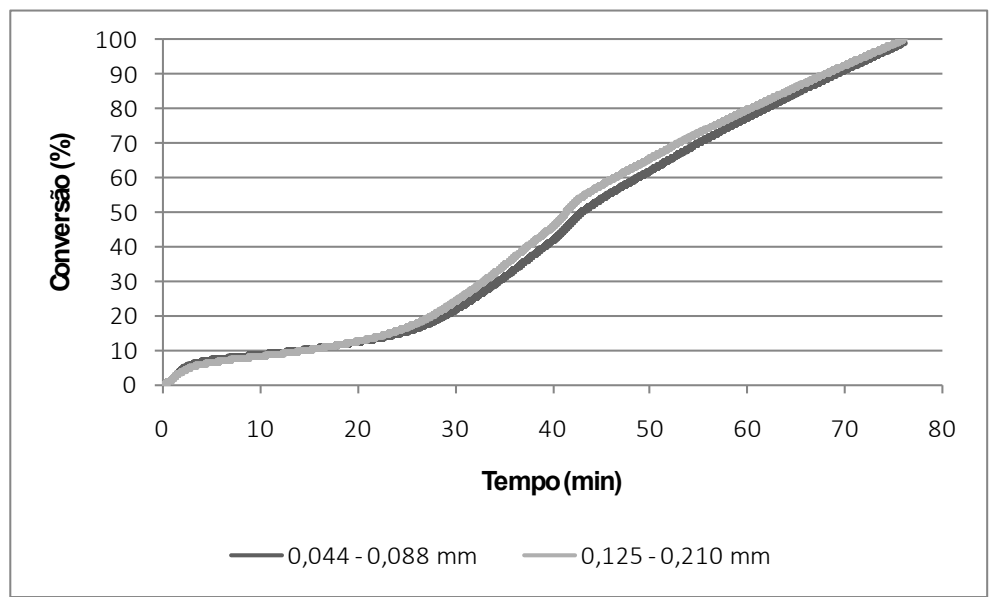

Fonte: os autores, 2017

Tabela 3 - Parâmetros cinéticos obtidos para as amostras

\begin{tabular}{|c|c|c|c|}
\hline \multicolumn{4}{|c|}{$0,044-0,088 \mathrm{~mm}$} \\
\hline Tempo (min) & Média A (1/min) & $\mathrm{E}(\mathrm{J} / \mathrm{mol})$ & $\mathrm{k}$ médio (1/min) \\
\hline $0-2,5$ & 0,02859 & 77,89502 & 0,02221 \\
\hline $2,5-25$ & 0,00549 & 121,67980 & 0,00506 \\
\hline $25-41,5$ & 0,02770 & 104,64131 & 0,02702 \\
\hline $41,5-53,6$ & 0,04414 & 111,95539 & 0,04333 \\
\hline \multicolumn{4}{|c|}{$0,125-0,210 \mathrm{~mm}$} \\
\hline Tempo (min) & Média A (1/min) & $\mathrm{E}(\mathrm{J} / \mathrm{mol})$ & $\mathrm{k}$ médio $(1 / \mathrm{min})$ \\
\hline $0-2,5$ & 0,02268 & 60,51575 & 0,01947 \\
\hline $2,5-20$ & 0,00547 & 120,51620 & 0,00501 \\
\hline $20-40$ & 0,02466 & 111,62293 & 0,02396 \\
\hline $40-53,6$ & 0,04677 & 101,31672 & 0,04599 \\
\hline
\end{tabular}

Fonte: os autores, 2017

No gráfico de TGA, a intersecção entre as curvas ocorre em torno dos 40 min de experimento. Como pode ser observada na Tabela 3, nesse ponto a energia de ativação (E) para a amostra com menor granulometria é menor, quando comparada a outra amostra, apresentando também valores superiores de fator pré-exponencial e velocidade média. Essa mudança está de acordo com as curvas analisadas anteriormente, nas quais a amostra com menor granulometria passa a ter uma perda de massa mais acentuada a partir desse ponto. Dessa forma, os dados cinéticos estão de acordo com os dados de TGA.

Dentre as comparações, a amostra que possui uma menor granulometria apresentou uma maior perda de massa. Segundo Stelmachowski et al. [14], pode ser observada uma dependência entre a reatividade e o tamanho da partícula, o qual os menores tamanhos de grão são mais reativos que os maiores, devido a uma maior capacidade de dispersão. Complementando, Ryu et al. [15] afirmam que partículas pequenas possuem maiores taxas de combustão e velocidade de ignição, e partículas maiores possuem uma maior concentração de $\mathrm{CO}_{2}$ por apresentarem menores taxas de combustão. Ainda, segundo os mesmos autores, partículas grandes são termicamente espessas com taxa de desvolatilização lenta e transferência de calor mais distribuída para as partículas próximas. 
Análise Termogravimétrica do Efeito da Granulometria do

Resíduo Metalúrgico na Cinética de Perda de Massa

\section{CONCLUSÕES}

A análise da perda de massa da mistura de biomassa de capim elefante, carvão vegetal e resíduo metalúrgico sob ação de um gás inerte foi realizada, em especial a ação da diferença no tamanho de grão, por meio de análise termogravimétrica. Dois diferentes tamanhos de grão foram utilizados e pode-se concluir que, para as condições de teste utilizadas, foi possível uma maior perda de massa, quando uma menor granulometria foi utilizada para o resíduo metalúrgico. Tais dados foram confirmados com os cálculos cinéticos realizados, os quais apresentaram valores de velocidade média maiores na faixa de 40 min de teste para a amostra com menor granulometria, o que justifica a maior perda de massa, a partir desse ponto.

\section{REFERÊNCIAS}

[1] QUINTINO, Manara Nogueira. Avaliação Da Reutilização De Resíduos Siderúrgicos Como Insumo Energético Para Uso Em Termoelétrica. 2017. Dissertação (Mestrado em Engenharia Mecânica) - UFF, Universidade Federal Fluminense, Volta Redonda, 2017.

[2] BAPTISTA Filho, B. "Relatório de Sustentabilidade 2014." Rio de Janeiro, Brasil: Instituto do Aço do Brasil. 2014.

[3] TAKANO, Cyro; CAPPOCHI, Jose Deodoro T; NASCIMENTO, Ramiro Conceição. A Reciclagem De Resíduos Siderúrgicos Sólidos. Seminário Nacional Sobre Reuso/Reciclagem De Resíduos Sólidos Industriais. São Paulo, USP. 13 p. 2000.

[4] ZOU, C.,ZHAO, J. Investigation Of Iron-Containing Powder On Coal Combustion Behavior. Journal Of The Energy Institute, 2016.

[5] LI, X. G., Ma, B. G., Xu, L., Luo, Z. T., \& Wang, K.. Catalytic effect of metallic oxides on combustion behavior of high ash coal. Energy \& Fuels, v. 21, n.5, p.2669-2672, 2007.

[6] GONG, Xuzhong; GUO, Zhancheng; WANG, Zhi. Reactivity of pulverized coals during combustion catalyzed by $\mathrm{CeO} 2$ and Fe2O3. Combustionand Flame, v. 157, n. 2, p. 351-356, 2010.

[7] HAN, Yunqing, Theoretical Study Of Thermal Analysis Kinetics. 2014. Dissertação (Mestrado em Engenharia Mecânica) - Universidade de Kentucky, Kentucky, 2014.

Engineering.

[8] MA, B. G., LI, X. G., XU, L., WANG, K., WANG, X. G. Investigation on catalyzed combustion of high ash coal by thermogravimetric analysis. Thermochimica Acta v. 445, n. 1, p. 19-22, 2006.

[9] YIN, K., ZHOU, Y. M., YAO, Q. Z., FANG, C., ZHANG, Z. W. Thermogravimetric analysis of the catalytic effect of metallic compounds on the combustion behaviors of coals. Reaction Kinetics, Mechanisms and Catalysis, v. 106, n. 2, p. 369-377, 2012.

[10] YU, L. Y.; LI, P. S. Thermogravimetric analysis of coal and sludge co-combustion with microwave radiation dehydration. Journal of the Energy Institute, v. 87, n. 3, p. 220-226, 2014. 
[11] CHENG, J., ZHOU, F., XUAN, X., LIU, J., ZHOU, J., CEN, K. Cascade chain catalysis of coal combustion by $\mathrm{Na}-\mathrm{Fe}-\mathrm{Ca}$ composite promoters from industrial wastes. Fuel, v.181, p. 820-826, 2016.

[12] CHENG, J., ZHOU, F., XUAN, X., LIU, J., ZHOU, J., CEN, K. Comparison of the catalytic effects of eight industrial wastes rich in $\mathrm{Na}, \mathrm{Fe}, \mathrm{Ca}$ and $\mathrm{Al}$ on anthracite coal combustion. Fuel, v. 187, p. 398-402, 2017.

[13] JIAN, D. I. N. G., LIU, Q. C., JIANG, L. J., LIU, G. Q., SHAN, R. E. N., JIAN, Y. A. N. G.; FEI, M. E. N. G. Thermal Behavior and Kinetics of Raw/Pyrolytic Wood and Coal Blends during Co-combustion Process. Journal of Iron and Steel Research, International, v. 23, n. 9, p. 917-923, 2016.

[14] STELMACHOWSKI, P., Kopacz, A., Legutko, P., Indyka, P., Wojtasik, M., Ziemiański, L.,\& Kotarba, A. The role of crystallite size of iron oxide catalyst for soot combustion. Catalysis Today. v. 257, p. 111116, 2015.

[15] RYU, C., YANG, Y. B., KHOR, A., YATES, N. E., SHARIFI, V. N., SWITHENBANK, J. Effect of fuel properties on biomass combustion: part I. Experiments-fuel type, equivalence ratio and particle size. Fuel. v. 85 , n. 7, p. 1039-1046, 2006. 